CARDIOVASCULAR MEDICINE

\title{
Infective endocarditis in a Finnish teaching hospital: a study on 326 episodes treated during 1980-2004
}

\author{
M Heiro, H Helenius, S Mäkilä, U Hohenthal, T Savunen, E Engblom, J Nikoskelainen, \\ $P$ Kotilainen

See end of article for authors' affiliations

Correspondence to: Dr Maijo Heiro,

Department of Medicine, Turku University Hospital Kiinamyllynkatu 4-8,

20520 Turku, Finland; maija.heiro@tyks.fi

Accepted 13 April 2006

Published Online First

27 April 2006

\begin{abstract}
Objectives: To evaluate potential changes of infective endocarditis (IE) in patients treated in a Finnish teaching hospital during the past 25 years.

Patients: 326 episodes of IE in 303 patients treated during 1980-2004 were evaluated for clinical characteristics and their changes over time.

Results: The mean age of the patients increased with time (from 47.2 to 54.5 years, $p=0.003$ ). Twentyfive (7.7\%) episodes were associated with intravenous drug use (IVDU), with a significant increase of these episodes after 1996 (from 0 to $19(20 \%), p<0.001$ ). Viridans streptococci were the most common causative agents of IE during 1980-1994, but after that Staphylococcus aureus was the most common pathogen ( $p=0.015)$. The proportion of IE of the aortic valve decreased during the study (from $30(49 \%)$ to $26(27 \%)$, whereas the proportions of mitral ( $11(18 \%)$ to $33(35 \%)$ and tricuspid valve IE $(0$ to $13(14 \%)$ increased correspondingly $(p=0.001)$. This was mainly due to more patients with IVDU. Chronic dialysis for renal failure as an underlying condition increased over time (from 0 to $7(7.4 \%$ ), $p=0.015$ ) but no other predisposing conditions changed. Complications such as neurological manifestations and heart failure did not change in frequency, but the incidence of lung emboli increased (from $0 \%$ to $10.5 \%$, $\mathrm{p}<0.001$ ); $83 \%$ of these emboli occurred in patients with IVDU. The proportion of patients requiring surgical treatment and mortality due to IE did not change.

Conclusions: During these 25 years, the causative agents, affected valves and complications of IE changed to some degree. These changes were mainly attributed to the increase of IVDU-associated IE. Except for the increase in age, the clinical presentation and outcome in non-addicts remained substantially unchanged.
\end{abstract}

linicians treating patients with infective endocarditis (IE) have faced a changing pattern of the disease over the past two to three decades. The risk factors, causative microorganisms and the age of the patients contracting the disease have changed. ${ }^{1-3}$ Mitral valve prolapse, degenerative valve disorders and prosthetic valves have replaced chronic rheumatic heart disease as the most common cardiac condition predisposing to IE in the occidental industrialised world. ${ }^{2}{ }^{3}$ Furthermore, the number of patients without previously diagnosed heart disease has increased. ${ }^{2}$ New risk factors, such as intravenous drug use (IVDU), long-term haemodialysis and nosocomial disease have emerged over the years, ${ }^{2}$ and the age of patients with non-IVDU endocarditis has increased. ${ }^{2}{ }^{3}$

The causative microorganisms are different today than before. Classically, the predominant causative agent has been the viridans streptococcus, leading to the subacute (lenta) type of endocarditis. ${ }^{4-7}$ In many recently described patient populations, the most frequently isolated microorganism has been Staphylococcus aureus, manifested clinically by acute endocarditis.$^{8-16}$ However, a few studies did not identify any changes in the clinical presentation or course of endocarditis. ${ }^{17-19}$ The differences between studies may be due to differences in race, genetic factors, modes of living, bacterial environment, etc, among populations.

Our previous studies on patients treated for IE in a Finnish teaching hospital from 1980 onwards have focused on the diagnostic classification and the presence of neurological manifestations, as well as the utility of serum $\mathrm{C}$ reactive protein in assessing the outcome of IE. ${ }^{20-22}$ In the present study, we set out to evaluate the clinical presentation and outcome of patients with IE treated in our hospital during the past 25 years.

\section{METHODS}

We reviewed the medical records of adult patients with a discharge diagnosis or postmortem findings of IE treated at the Turku University Hospital, Turku, Finland, between 1980 and 2004. The hospital is a 1000-bed teaching facility with a cardiothoracic surgical department, serving as a tertiary referral centre for the southwestern part of the country and, in addition, providing primary care for infectious diseases in an area of about 200000 inhabitants. Cases of endocarditis were included in this study if they met the Duke criteria for definite or possible IE. ${ }^{23}$

\section{Patients}

For each patient, data were collected on age, sex, predisposing heart disease and other predisposing conditions, such as IVDU, preceding surgical and endoscopic procedures, various underlying diseases, alcohol misuse and infections of the oral cavity or skin. The occurrence of fever and other classic clinical symptoms of IE, and the duration of symptoms and administration of per oral antimicrobial treatment before the admission were reviewed. The patients were evaluated for the results of blood cultures, involved valves, echocardiographic findings and the presence of new murmurs of valvular regurgitation. Neurological complications, peripheral emboli, heart failure, cardiac surgery and death due to IE were recorded from the onset of symptoms to three months after the admission of a patient to hospital.

Abbreviations: IE, infective endocarditis; IVDU, intravenous drug use; $\mathrm{TOE}$, transoesophageal echocardiography; TTE, transthoracic echocardiography 
Table 1 Characteristics of 326 episodes of infective endocarditis analysed together and separately in five-year periods

\begin{tabular}{|c|c|c|c|c|c|c|c|}
\hline & 1980-2004 & $1980-4$ & $1985-9$ & $1990-4$ & $1995-9$ & $2000-4$ & p Value \\
\hline No of episodes & 326 & 61 & 45 & 65 & 60 & 95 & $<0.001$ \\
\hline Sex & & & & & & & $0.336^{*}$ \\
\hline Male & $234(71.8 \%)$ & $48(78.7 \%)$ & $30(66.7 \%)$ & $43(66.2 \%)$ & $47(78.3 \%)$ & $66(69.5 \%)$ & \\
\hline Female & $92(28.2 \%)$ & $13(21.3 \%)$ & $15(33.3 \%)$ & $22(33.9 \%)$ & $13(21.7 \%)$ & $29(30.5 \%)$ & \\
\hline Mean (SD) age (years) & $54.4(17.3)$ & $47.2(17.6)$ & $54.0(16.4)$ & $59.4(11.7)$ & $56.3(17.3)$ & $54.5(19.5)$ & 0.003 \\
\hline Affected valves & & & & & & & $0.001^{*}$ \\
\hline Aortic & $113(34.7 \%)$ & $30(49.2 \%)$ & $15(33.3 \%)$ & $26(40.0 \%)$ & $16(26.7 \%)$ & $26(27.4 \%)$ & \\
\hline Mitral & $96(29.5 \%)$ & $11(18.0 \%)$ & $10(22.2 \%)$ & $22(33.9 \%)$ & $20(33.3 \%)$ & $33(34.7 \%)$ & \\
\hline Tricuspid & $18(5.5 \%)$ & $0 \%$ & $1(2.2 \%)$ & $0 \%$ & $4(6.7 \%)$ & $13(13.7 \%)$ & \\
\hline Two native valves & $32(9.8 \%)$ & $6(9.8 \%)$ & $6(13.3 \%)$ & $3(4.6 \%)$ & $9(15.0 \%)$ & $8(8.4 \%)$ & \\
\hline Prosthetic valve(s) & $67(20.6 \%)$ & $14(23.0 \%)$ & $13(28.9 \%)$ & $14(21.5 \%)$ & $11(18.3 \%)$ & $15(15.8 \%)$ & \\
\hline Blood cultures & & & & & & & $0.163^{*}$ \\
\hline Staphylococcus aureus & $75(23.0 \%)$ & $7(11.5 \%)$ & $7(15.6 \%)$ & $12(18.5 \%)$ & $18(30.0 \%)$ & $31(32.6 \%)$ & \\
\hline Coagulase-negative staphylococci & $31(9.5 \%)$ & $7(11.5 \%)$ & $3(6.7 \%)$ & $9(13.9 \%)$ & $4(6.7 \%)$ & $8(8.4 \%)$ & \\
\hline Viridans streptococci & $67(20.6 \%)$ & $11(18.0 \%)$ & $9(20.0 \%)$ & $14(21.5 \%)$ & $14(23.3 \%)$ & $19(20.0 \%)$ & \\
\hline Enterococcus faecalis & $28(8.6 \%)$ & $10(16.4 \%)$ & $5(11.1 \%)$ & $3(4.6 \%)$ & $4(6.7 \%)$ & $6(6.3 \%)$ & \\
\hline Streptococcus pneumoniae & $11(3.4 \%)$ & $2(3.3 \%)$ & $1(2.2 \%)$ & $2(3.1 \%)$ & $2(3.3 \%)$ & $4(4.2 \%)$ & \\
\hline Other & $25(7.7 \%)$ & $6(9.8 \%)$ & $2(4.4 \%)$ & $3(4.6 \%)$ & $4(6.7 \%)$ & $10(10.5 \%)$ & \\
\hline Negative & $89(27.3 \%)$ & $18(29.5 \%)$ & $18(40.0 \%)$ & $22(33.9 \%)$ & $14(23.3 \%)$ & $17(17.9 \%)$ & \\
\hline Echocardiographic findings & & & & & & & $<0.001^{*}$ \\
\hline Major & $221(67.8 \%)$ & $35(57.4 \%)$ & $22(48.9 \%)$ & $44(67.7 \%)$ & $46(76.7 \%)$ & $74(77.9 \%)$ & \\
\hline Minor & $60(18.4 \%)$ & $8(13.1 \%)$ & $13(28.9 \%)$ & $17(26.2 \%)$ & $7(11.7 \%)$ & $15(15.8 \%)$ & \\
\hline No findings & 45 (13.8\%) & $18(29.5 \%)$ & $10(22.2 \%)$ & $4(6.2 \%)$ & 7 (11.7\%) & $6(6.3 \%)$ & \\
\hline
\end{tabular}

The clinical characteristics of the episodes were analysed both collectively and separately in five-year study periods to define the potential changes in the clinical presentation of patients with IE treated in our hospital since 1980.

\section{Statistical analysis}

Differences between the five-year time periods in distributions of the categorical variables were statistically tested by Pearson's $\chi^{2}$ test. Exact $p$ values were calculated in the case of small frequencies. The difference between time periods in the mean age was tested with one-way analysis of variance. The difference between blood culture groups and the change over time in the duration of the symptoms before admission were analysed by the Kruskal-Wallis test.

Values of $\mathrm{p}<0.05$ were considered significant. Statistical calculations were performed with SAS System for Windows V9.1.3/2004 (SAS Institute, Cary, North Carolina, USA).

\section{RESULTS}

During the years 1980-2004, a total of 326 episodes in 303 patients fulfilled the Duke criteria for definite or possible IE. Of these episodes, 224 were designated as definite IE and 102 as possible IE. Tables 1 and 2 present the characteristics and predisposing conditions of the patients.

The mean duration of the symptoms before admission to hospital was 16.7 (SD 31.3) days. The mean duration of symptoms before admission was significantly shorter for the $S$ aureus episodes than for the other episodes combined (6.6 days $v 19.7$ days, $\mathrm{p}<0.0001)$. Figure 1 presents the most common clinical symptoms of the patients. Patients had high fever of $\geqslant 38^{\circ} \mathrm{C}$ in 244 episodes and no fever in 22 episodes. A new regurgitant valvular murmur was heard in $164(50.3 \%)$ episodes; in $40(12.3 \%)$ episodes no murmur was heard. Enlargement of the spleen was detected by palpation or ultrasound in $38(11.7 \%)$ episodes. Dental abscesses or

Table 2 Predisposing conditions of patients in 326 episodes of infective endocarditis analysed together and separately in fiveyear periods

\begin{tabular}{|c|c|c|c|c|c|c|c|}
\hline & 1980-2004 & $1980-4$ & $1985-9$ & $1990-4$ & $1995-9$ & $2000-4$ & p Value \\
\hline No of episodes & 326 & 61 & 45 & 65 & 60 & 95 & \\
\hline Cardiac conditions & & & & & & & $0.491^{*}$ \\
\hline Acquired valvular disease & $75(23.0 \%)$ & $17(27.9 \%)$ & $8(17.8 \%)$ & $15(23.1 \%)$ & $13(21.7 \%)$ & $22(23.2 \%)$ & \\
\hline Prosthetic valves & $67(20.6 \%)$ & $14(23.0 \%)$ & $13(28.9 \%)$ & $14(21.5 \%)$ & $11(18.3 \%)$ & $15(15.8 \%)$ & \\
\hline Bicuspid aortic valve & $38(11.7 \%)$ & $9(14.8 \%)$ & $6(13.3 \%)$ & $7(10.8 \%)$ & $6(10.0 \%)$ & $10(10.5 \%)$ & \\
\hline Mitral valve prolapse & $33(10.1 \%)$ & $2(3.3 \%)$ & $4(8.9 \%)$ & $11(16.9 \%)$ & $7(11.7 \%)$ & $9(9.5 \%)$ & \\
\hline Congenital heart disease & $10(3.1 \%)$ & $3(4.9 \%)$ & $2(4.4 \%)$ & $3(4.6 \%)$ & $0 \%$ & $2(2.1 \%)$ & \\
\hline No underlying cardiac condition & $103(31.6 \%)$ & $16(26.2 \%)$ & $12(26.7 \%)$ & $15(23.1 \%)$ & $23(38.3 \%)$ & $37(39.0 \%)$ & \\
\hline Alcohol misuse & $29(8.9 \%)$ & $5(8.2 \%)$ & $4(8.9 \%)$ & $7(10.8 \%)$ & $8(13.3 \%)$ & $5(5.3 \%)$ & 0.505 \\
\hline Intravenous drug use & $25(7.7 \%)$ & $0 \%$ & $0 \%$ & $0 \%$ & $6(10.0 \%)$ & $19(20.0 \%)$ & $<0.001$ \\
\hline $\begin{array}{l}\text { Dialysis } \\
\text { Underlyina diseases }\end{array}$ & $10(3.1 \%)$ & $0 \%$ & $0 \%$ & $0 \%$ & $3(5.0 \%)$ & $7(7.4 \%)$ & 0.015 \\
\hline Diabetes & $31(9.5 \%)$ & $2(3.3 \%)$ & $5(11.1 \%)$ & $9(13.9 \%)$ & $7(11.7 \%)$ & $8(8.4 \%)$ & 0.313 \\
\hline Collagen disease & $22(6.8 \%)$ & $6(9.8 \%)$ & $2(4.4 \%)$ & $3(4.6 \%)$ & $4(6.7 \%)$ & $7(7.4 \%)$ & 0.785 \\
\hline Malignancy & $15(4.6 \%)$ & $2(3.3 \%)$ & $0 \%$ & $2(3.1 \%)$ & $2(3.3 \%)$ & $9(9.5 \%)$ & 0.078 \\
\hline Dental infections & $107(32.8 \%)$ & $12(19.7 \%)$ & $17(37.8 \%)$ & $24(36.9 \%)$ & $17(28.3 \%)$ & $37(38.9 \%)$ & 0.244 \\
\hline Prior surgical procedures & $51(15.7 \%)$ & $17(27.9 \%)$ & $5(11.1 \%)$ & $7(10.8 \%)$ & $7(11.7 \%)$ & $15(15.8 \%)$ & $0.085^{*}$ \\
\hline Dental & $29(8.9 \%)$ & $10(16.4 \%)$ & $2(4.4 \%)$ & $7(10.8 \%)$ & $2(3.3 \%)$ & $8(8.4 \%)$ & \\
\hline Urological & $8(2.5 \%)$ & $3(4.9 \%)$ & $1(2.2 \%)$ & $0 \%$ & $1(1.7 \%)$ & $3(3.2 \%)$ & \\
\hline Gastrointestinal & $6(1.8 \%)$ & $0 \%$ & $2(4.4 \%)$ & $0 \%$ & $2(3.3 \%)$ & $2(2.1 \%)$ & \\
\hline Other & $8(2.5 \%)$ & $4(6.6 \%)$ & $0 \%$ & $0 \%$ & 2 (3.3\%) & $2(2.1 \%)$ & \\
\hline
\end{tabular}

${ }^{*} p$ value for overall group differences. 


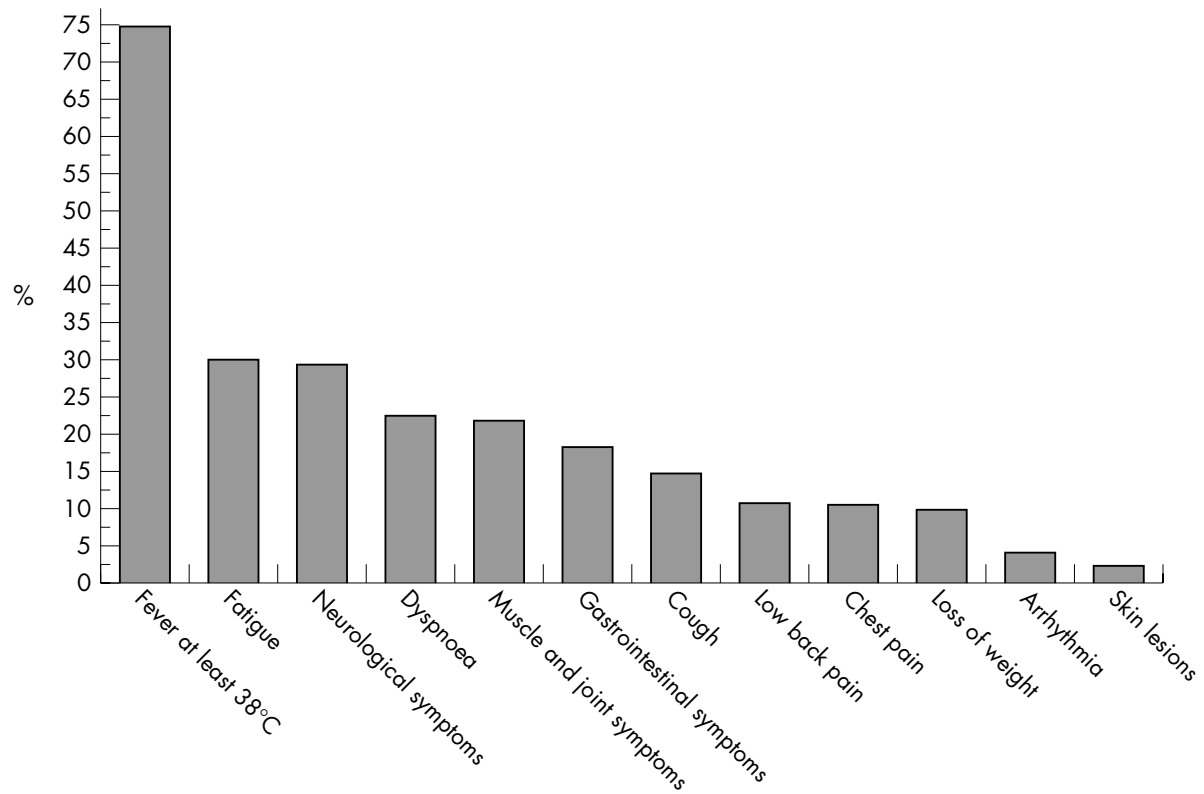

Figure 1 General symptoms in 326 episodes of infective endocarditis in 303 patients treated at the Turku University Hospital during 1980-2004.

periodontitis preceded IE in 107 episodes, and in 36 episodes skin lesions were the potential sources of infection.

Valve surgery was needed within three months after the diagnosis in $94(28.8 \%)$ episodes (table 3$)$. Of the 94 cardiac operations, $43(45.7 \%)$ were performed within 14 days after admission, $16(17.0 \%)$ within 15-30 days after admission and $35(37.2 \%)$ between one and three months of admission. Mechanical prosthetic valves were used in 75 episodes and bioprosthetic valves in 12 episodes. Mitral valvuloplasty was performed in four episodes and other operations in three episodes. The causative agents in the patients undergoing mitral valvuloplasty were viridans streptococci $(\mathrm{n}=2), S$ aureus $(\mathrm{n}=1)$ and $S$ epidermidis $(\mathrm{n}=1)$. In all of these patients, mitral valvuloplasty was performed in the acute phase of the disease during antimicrobial treatment.

Altogether $43(13.2 \%)$ patients died of IE within three months of admission: 23 of them within 30 days of admission and 20 between 1-3 months of admission. Mortality was $12.5 \%$ in conservatively treated episodes and $14.9 \%$ in surgically treated episodes.

\section{Changes of clinical characteristics over the five-year periods after 1980}

When analysed separately in five-year periods, the number of episodes of IE were shown to increase with time (table 1). The larger number of IE episodes seen during the last study period was partly due to the emergence of episodes associated with IVDU. The first such case was diagnosed in 1996, after which the increase of IVDU-associated IE was significant (table 2).

The sex distribution of the patients did not change between time periods (table 1). The mean age of the patients with IE increased significantly, from 47.2 years during $1980-4$ to 54.5 years during 2000-4. During the last five-year period, the

Table 3 Complications, mode of treatment and outcome of 326 episodes of infective endocarditis analysed together and separately in five-year periods

\begin{tabular}{|c|c|c|c|c|c|c|c|}
\hline & 1980-2004 & $1980-4$ & 1985-9 & $1990-4$ & 1995-9 & $2000-4$ & p Value \\
\hline No of episodes & 326 & 61 & 45 & 65 & 60 & 95 & \\
\hline Neurological complications & $86(26.4 \%)$ & $15(24.6 \%)$ & $13(28.9 \%)$ & $16(24.6 \%)$ & $19(31.7 \%)$ & $23(24.2 \%)$ & $0.672^{*}$ \\
\hline Embolic brain infarction & $27(8.3 \%)$ & $5(8.2 \%)$ & $2(4.4 \%)$ & $3(4.6 \%)$ & $8(13.3 \%)$ & $9(9.5 \%)$ & \\
\hline Transient ischaemic attack & $16(4.9 \%)$ & $3(4.9 \%)$ & $2(4.4 \%)$ & $4(6.2 \%)$ & $5(8.3 \%)$ & $2(2.1 \%)$ & \\
\hline Meningitis & $5(4.6 \%)$ & $4(6.6 \%)$ & $3(6.7 \%)$ & $2(3.1 \%)$ & $1(1.7 \%)$ & $5(5.3 \%)$ & \\
\hline Brain haemorrhage & $6(1.8 \%)$ & $1(1.6 \%)$ & $1(2.2 \%)$ & $0 \%$ & $1(1.7 \%)$ & $3(3.2 \%)$ & \\
\hline Others & $22(6.8 \%)$ & $2(3.3 \%)$ & $5(11.1 \%)$ & $7(10.8 \%)$ & $4(6.7 \%)$ & $4(4.2 \%)$ & \\
\hline Emboli outside CNS & $102(31.3 \%)$ & $17(27.9 \%)$ & $13(28.9 \%)$ & $15(23.1 \%)$ & $24(40.0 \%)$ & $33(34.7 \%)$ & 0.274 \\
\hline HF & $178(54.6 \%)$ & $38(62.3 \%)$ & $24(53.3 \%)$ & $39(60.0 \%)$ & $32(53.3 \%)$ & $45(47.4 \%)$ & 0.368 \\
\hline Mode of treatment within 3 months & & & & & & & 0.943 \\
\hline Surgical & $94(28.8 \%)$ & $20(32.8 \%)$ & $12(26.7 \%)$ & $18(27.7 \%)$ & $16(26.7 \%)$ & $28(29.5 \%)$ & \\
\hline Conservative & $232(71.2 \%)$ & $41(67.2 \%)$ & $33(73.3 \%)$ & $47(72.3 \%)$ & $44(73.3 \%)$ & $67(70.5 \%)$ & \\
\hline Indications for surgery & $94(28.8 \%)$ & $20(32.8 \%)$ & $12(26.7 \%)$ & $18(27.7 \%)$ & $16(26.7 \%)$ & $28(29.5 \%)$ & $0.265^{*}$ \\
\hline Congestive HF & 55 (16.9\%) & $12(19.7 \%)$ & $7(15.6 \%)$ & $7(10.8 \%)$ & 11 (18.3\%) & 18 (18.9\%) & \\
\hline Valvular regurgitation without $\mathrm{HF}$ & $43(13.2 \%)$ & $14(23.0 \%)$ & $6(13.3 \%)$ & $12(18.5 \%)$ & $4(6.7 \%)$ & $7(7.4 \%)$ & \\
\hline $\begin{array}{l}\text { Dehiscence of prosthetic valve } \\
\text { without HF }\end{array}$ & $10(3.1 \%)$ & $1(1.6 \%)$ & $2(4.4 \%)$ & $4(6.2 \%)$ & $0 \%$ & $3(3.2 \%)$ & \\
\hline Repeated emboli & $5(1.5 \%)$ & $1(1.6 \%)$ & $1(2.2 \%)$ & $2(3.1 \%)$ & $1(1.7 \%)$ & $0 \%$ & \\
\hline Intractable infection & $6(1.8 \%)$ & $1(1.6 \%)$ & $0 \%$ & $1(1.5 \%)$ & $1(1.7 \%)$ & $3(3.2 \%)$ & \\
\hline Outcome of infective endocarditis & & & & & & & 0.781 \\
\hline Death & $43(13.2 \%)$ & $9(14.8 \%)$ & $5(11.1 \%)$ & $6(9.2 \%)$ & $8(13.3 \%)$ & $15(15.8 \%)$ & \\
\hline Survival & $283(86.8 \%)$ & $52(85.2 \%)$ & $40(88.9 \%)$ & $59(90.8 \%)$ & $52(86.7 \%)$ & 80 (84.2\%) & \\
\hline
\end{tabular}

* $p$ value for overall group differences.

CNS, central nervous system; HF, heart failure. 
mean age of the patients with no IVDU was 62.0 years. The proportions of mitral and tricuspid valve IE increased with time, whereas the proportions of aortic and prosthetic valve IE decreased. These trends were not significant when the patients with IVDU were excluded from the analyses. The overall distribution of the causative agents did not change significantly. During 1980-1994, viridans streptococci were the most common causative agents, after which $S$ aureus was the most common. When analysed separately for the $S$ aureus episodes, culture-negative episodes and the rest of the episodes combined, the role of $S$ aureus as the causative agent increased during the study, whereas the proportion of culture-negative episodes decreased $(p=0.015)$. When the patients with IVDU-associated IE were excluded, the increase in the $S$ aureus aetiology vanished and, in fact, decreased, with $24.1 \%$ of the episodes between 1995 and 1999, and $22.4 \%$ between 2000 and 2004 being caused by this pathogen.

Transthoracic echocardiography (TTE) was performed in all episodes of IE, followed by transoesophageal echocardiography (TOE) in 184 episodes. The proportion of episodes with major echocardiographic findings (that is, vegetation, abscess and dehiscence of a prosthetic valve) increased concurrently with a decrease of episodes without new echocardiographic findings (table 1). The proportions of patients having only TTE, or both TTE and TOE, varied during the different study periods: the proportion of patients having TOE was $4.9 \%$ during $1980-5,33.3 \%$ during $1985-9,76.9 \%$ during 1990-4, 88.3\% during $1995-9$ and $66.3 \%$ during $2000-$ $4(\mathrm{p}<0.001)$. A major echocardiographic criterion was shown by TTE in 30 (93.6\%) of the 32 episodes between 2000 and 2004 in which only TTE was performed.

Chronic dialysis as an underlying condition increased significantly during the study (table 2). Predisposing cardiac conditions and other underlying diseases of the patients did not change. The frequency of dental infections and dental or other surgical interventions preceding IE also did not change. The duration of the symptoms before admission did not change over time $(\mathrm{p}=0.247)$.

The incidence of neurological complications and emboli outside the central nervous system remained unchanged (table 3) but, when analysed separately, the incidence of pulmonary emboli increased $(0 \% v 10.5 \%, \mathrm{p}<0.001)$. Of the lung emboli, $83.3 \%$ (10 of 12 ) occurred in patients with IVDU. The frequency of patients developing heart failure as a complication of IE remained on the same level. Also the proportion of patients requiring surgical treatment for IE within three months after admission remained unchanged, as did the incidence of congestive heart failure as the main indication for surgery. Total IE mortality and mortality of the conservatively or surgically treated patients did not change when analysed separately.

Fever $\geqslant 38^{\circ} \mathrm{C}$ was the most common clinical symptom of IE throughout the study, occurring in $67.4-81.6 \%$ of the patients during the different time periods.

\section{Episodes of IVDU-associated IE}

Twenty-five $(7.7 \%)$ of the episodes of IE were in patients with IVDU. The mean age of these patients was 24.2 (SD 3.4) years (range 19-33 years). Of the 25 episodes, 21 (84.0\%) were in male patients and four $(16.0 \%)$ in female patients. The mean duration of symptoms before admission was 4.8 (SD 6.9) days (range $<1$ to 30 days). The most common causative agent in these patients was $S$ aureus accounting for $20(80.0 \%)$ episodes. Tricuspid valve was affected in 15 (60.0\%) episodes, the mitral valve in five $(20.0 \%)$ episodes, two native valves in three $(12.0 \%)$ episodes and prosthetic valves in two $(8.0 \%)$ episodes. Neurological complications developed in six $(24.0 \%)$ episodes and emboli outside the central nervous system in $14(56.0 \%)$ episodes; on 10 occasions, lungs were involved. Three $(12.0 \%)$ patients were treated surgically for IE. IE mortality within three months after the diagnosis was $4.0 \%$ (one patient) in IVDU-associated episodes of IE.

\section{Dialysis-associated episodes of IE}

Eight patients with 10 episodes of IE were on chronic dialysis for renal failure. The mean age of these patients was 64.0 (SD 11.2) years (range 46-80 years); men and women were equally affected. Eight episodes were associated with haemodialysis and two episodes with peritoneal dialysis. Native mitral valves were involved in seven $(70 \%)$ and native aortic valves in three $(30 \%)$ of these episodes. All patients had a previously recognised valvular disorder predisposing to IE. Echocardiography showed valvular vegetation in all episodes, in $60 \%$ of them only by TOE. The causative agents in dialysis-associated episodes of IE were coagulase-negative staphylococci $(\mathrm{n}=4), S$ aureus $(\mathrm{n}=1)$ and Enterococcus faecalis $(\mathrm{n}=1)$, and four episodes were culture negative. IE was complicated by congestive heart failure in eight $(80 \%)$ cases and by embolic events in five $(50 \%)$. Two patients required valve surgery due to heart failure during the acute phase of IE. One of them died on the first postoperative day and the other survived. In these dialysis-associated episodes, IE mortality within three months after the diagnosis was $40 \%$ (four of 10).

\section{Episodes of IE in elderly patients}

A total of $65(19.9 \%)$ episodes occurred in patients $\geqslant 70$ years of age: $37(56.9 \%)$ in male patients and $28(43.1 \%)$ in female patients. Native valves were affected in $55(84.6 \%)$ episodes and prosthetic valves in $10(15.4 \%)$. The mitral valve was the most commonly affected native valve $(\mathrm{n}=29)$, followed by the aortic valve $(\mathrm{n}=21)$. The causative agents were $S$ aureus $(\mathrm{n}=16)$, E faecalis $(\mathrm{n}=12)$, viridans streptococci $(\mathrm{n}=7)$, coagulase-negative staphylococci $(\mathrm{n}=6)$, and other pathogens (4); 20 episodes were culture negative. IE was complicated by neurological manifestations in 11 (16.9\%) episodes and by peripheral emboli in 22 (33.8\%). Surgical treatment was required in six $(9.2 \%)$ episodes. In these episodes affecting elderly patients, IE mortality within three months after the diagnosis was $16.9 \%$ (11 of 65).

\section{Patients with repeated episodes of IE}

Seventeen patients had two episodes of IE and three patients had three episodes of IE. Of these 43 episodes in 20 patients, $33(76.7 \%)$ were in men and $10(23.3 \%)$ in women, the mean age of the patients being 45.8 (SD 16.5) years (range 22-75 years). The mean time elapsed between the first episode and the recurrence(s) was 45 (SD 46) months (range 2-145 months). The causative agents in these patients with repeated episodes were coagulase-negative staphylococci $(\mathrm{n}=10), S$ aureus $(\mathrm{n}=9)$, viridans streptococci $(\mathrm{n}=7)$, E faecalis $(\mathrm{n}=1)$ and other pathogens $(\mathrm{n}=2)$; 14 episodes were culture negative. The most common predisposing cardiac condition was the presence of a prosthetic valve (20 episodes). IE was complicated by neurological manifestations in $27.9 \%$ ( 12 of 43 ) and by peripheral emboli in $23.3 \%$ ( 10 of 43 ) of the episodes. Valve surgery was required in $27.9 \%$ ( 12 of 43 ) of the episodes. IE mortality within three months after the diagnosis was 7\% (three patients).

\section{DISCUSSION}

In this study we analysed 326 episodes of IE treated in a Finnish teaching hospital over 25 years. Our purpose was to assess whether our patients population would also manifest changes commonly reported to have occurred in the clinical presentation of endocarditis. We consider the emergence and significant increase of IVDU-associated episodes of IE after 1996 to be the most important finding of the study. These 
episodes constituted only $8 \%$ of all episodes but as much as $20 \%$ of the episodes between 2000 and 2004. Even this is a small proportion compared with some recently published reports, in which more than half of the patients have been drug addicts. ${ }^{11}$ Consistent with previous studies, ${ }^{8}$ chronic dialysis as a condition predisposing a patient to IE also increased during this study, but the total number of the disease episodes in these patients was small, having a minor effect on the clinical variables evaluated.

In many recently described patient populations, $S$ aureus has surpassed viridans streptococci as the most common causative agent of IE. ${ }^{8}{ }^{12}{ }^{14-16}$ We saw the same development in the episodes of IE analysed here, as viridans streptococci were the predominant pathogens during 1980-94, but after that the proportion of $S$ aureus increased significantly and it became the most common. In our patients, this increase was mainly due to the emergence of IVDU-associated endocarditis caused by $S$ aureus. If the patients with IVDU were excluded from the analysis, the proportions of viridans streptococci and $S$ aureus were practically equal during the last five-year period ( $22 \%$ and $21 \%$, respectively). These results underscore the important role oral streptococci still have as causative agents of endocarditis in our area.

The aortic valve was the predominant site of infection in the whole patient population. However, the proportions of aortic valve and prosthetic valve IE decreased with time, whereas the proportions of mitral and tricuspid valve IE increased. This was due to the increased number of patients with IVDU-associated IE: patients with no IVDU did not have these changes in proportions. These findings lead to the conclusion that the changes in causative agents and affected valves observed in this study were associated with the emergence of IVDU, as these characteristics remained substantially unchanged in the patients with no IVDU. In this respect, the present study corroborates a few others, in which the clinical presentation or course of endocarditis remained unchanged during recent decades. ${ }^{17-19}$ One of these studies ${ }^{18}$ was a small survey of the Minnesota population and two others come from European centres. ${ }^{17} 19$ A common feature of these studies is that they found no or only a low incidence of IVDU-associated IE. In contrast, several other recent studies from European countries have reported important changes in the disease characteristics and epidemiology of IE. ${ }^{14} 1624$

In our patients, IE mortality during three months after the diagnosis was $13.2 \%$, and it did not change over time. This is in agreement with previous studies from the 1990s and 2000s with in-hospital IE mortalities between $10-24 \%{ }^{13}{ }^{1724-29}$ In a few series, mortality has tended to decrease, and this has been attributed either to decreased operative mortality ${ }^{19}$ or to an increased rate of successful early valve surgery. ${ }^{24}$ During the whole study period, mortality remained slightly higher in our surgically treated patients than in those treated conservatively, and the proportion of patients requiring surgery did not change. Similarly, the incidence of neurological manifestations, peripheral emboli and heart failure as complications of IE did not change. Only the incidence of lung emboli increased, but these complications occurred mainly in the patients with IVDU.

We did not find any major change in the predisposing heart diseases of our patients between time periods. The most common predisposing cardiac condition was acquired valvular disease, followed by a prosthetic heart valve. Although mitral valve prolapse is now thought to be a major cardiac abnormality predisposing to IE, ${ }^{30}$ only $10 \%$ of our patients had this condition. Several earlier papers from the 2000s reported an increase in the proportion of IE in patients with no predisposing heart disease. ${ }^{24}$ We also observed this trend, with an increase of such episodes from $26 \%$ to $39 \%$ between the first and last study periods, but the change was not significant.

IE is increasingly considered a disease of old people. ${ }^{31}$ This concept is supported by the results of the present study showing a significant increase (from 47 to 55 years) in the mean age of the patients between the first and last five-year period. The increase was even more conspicuous among patients with no IVDU, whose mean age was 62 years during the last period.

The proportion of episodes with major echocardiographic findings increased with time, whereas the proportion of episodes without new abnormalities decreased. This is evidently associated with major advancement in echocardiographic techniques and skills during the long study period. TOE is now the preferred method in the evaluation of patients with suspected endocarditis, but only a small proportion of our patients had TOE during the first five-year period. Subsequently, the proportion of TOE increased massively, with $88 \%$ of the patients with IE between 1995 and 1999 having this procedure. Surprisingly, TOE was performed in only $66 \%$ of the episodes of IE during the last five-year period. Still, this is reasonable considering that in the great majority (94\%) of the episodes with no TOE, major echocardiographic findings were already evident on TTE, probably due to better accuracy of detecting vegetations by TTE with modern echocardiography equipment.

\section{Study strengths and limitations}

The present work provides a good opportunity to evaluate recent trends in the clinical presentation and outcome of IE, as a large number of patients treated in one institution were analysed and the duration of the study was long. Yet the possible influence of referral bias on our results cannot be totally excluded because, besides providing primary care for infectious diseases, ${ }^{32}$ our hospital is a tertiary care institute. Acute infections are also treated in four regional hospitals in our area, but the practice has been to refer all patients with suspected or diagnosed IE to our infectious diseases unit. During these years, a few elderly patients with subacute uncomplicated IE were treated in regional hospitals, whereas all patients with acute IE or prosthetic valve IE were invariably referred, as well as all of those with subacute IE who developed complications, regardless of their age. According to our experience, no major changes in the referral policy were made during the study period. The fact that our patient population includes both severe and mild cases of IE, thus representing the full spectrum of the disease, is a further strength of this work.

\section{Conclusions}

The emergence of IVDU-associated episodes of IE was the major change in the clinical pattern of IE among the patients treated in our hospital. The significant increase of these episodes after 1996 explained all the changes in causative agents, affected valves and complications observed during the study period. Except for the increase in age, the clinical presentation and outcome of IE in the patients with no IVDU remained substantially unchanged during 25 years of observation.
Authors' affiliations
M Heiro, U Hohenthal, E Engblom, J Nikoskelainen, P Kotilainen,
Department of Medicine, Turku University Hospital, Turku, Finland
T Savunen, Department of Surgery, Turku University Hospital, Turku, Finland
H Helenius, S Mäkilä, Department of Biostatistics, University of Turku, Turku, Finland
None of the authors received any financial support for the study.
Competing interests: None declared. 


\section{REFERENCES}

1 Bayer AS. Infective endocarditis. Clin Infect Dis 1993;17:313-22.

2 Moreillon P, Que Y-A. Infective endocarditis. Lancet 2004;363:139-49.

3 Mylonakis E, Calderwood SB. Infective endocarditis in adults. N Engl J Med 2001;345:1318-30.

4 Bayliss R, Clarke C, Oakley CM, et al. The microbiology and pathogenesis of infective endocarditis. Br Heart J 1983;50:513-9.

5 Garvey GJ, Neu HC. Infective endocarditis: an evolving disease. Medicine 1978:57:105-27.

6 Griffin MR, Wilson WR, Edwards WD, et al. Infective endocarditis: Olmsted County, Minnesota, 1950 through 1981. JAMA 1985;254:1199-202.

7 Von Reyn CF, Levy BS, Arbeit RD, et al. Infective endocarditis: an analysis based on strict case definitions. Ann Intern Med 1981;94:505-18.

8 Cabell CH, Jollis JG, Peterson GE, et al. Changing patient characteristics and the effect on mortality in endocarditis. Arch Intern Med 2002;162:90-4.

9 Fowler VG, Miro JM, Hoen B, et al. Staphylococcus aureus endocarditis: a consequence of medical progress. JAMA 2005;293:3012-21.

10 Hogevik H, Olaison L, Andersson R, et al. Epidemiologic aspects of infective endocarditis in an urban population. Medicine 1995;74:324-39.

11 Siddiq S, Missri J, Silverman DI. Endocarditis in an urban hospital in the 1990s. Arch Intern Med 1996;156:2454-8.

12 Tornos $\mathbf{P}$, lung B, Permanyer-Miralda G, et al. Infective endocarditis in Europe: lessons from the Euro heart survey. Heart 2005;91:571-5.

13 Watanakunakorn C, Burkert T. Infective endocarditis at a large community teaching hospital, 1980-1990: a review of 210 episodes. Medicine (Baltimore) 1993;72:90-102.

14 Cecchi E, Forno D, Imazio M, et al. New trends in the epidemiological and clinical features of infective endocarditis: results of a multicenter prospective study. Ital Heart J 2004;5:249-56.

15 Ferreiros E, Nacinovich F, Casabé JH, et al. Epidemiologic, clinical, and microbiologic profile of infective endocarditis in Argentina: a national survey. The Endocarditis Infecciosa en la República Argentina-2 (EIRA-2) Study. Am Heart J 2006;151:545-52.

16 Loupa C, Mavroidi N, Boutsikakis I, et al. Infective endocarditis in Greece: a changing profile. Epidemiological, microbiological and therapeutic data. Clin Microbiol Infect 2004; 10:556-61.

17 Netzer ROM, Zollinger E, Seiler C, et al. Infective endocarditis: clinical spectrum, presentation and outcome. An analysis of 212 cases 1980-1995. Heart 2000;84:25-30.
18 Tleyjeh IM, Steckelberg JM, Murad HS, et al. Temporal trends in infective endocarditis: a population-based study in Olmsted County, Minnesota. JAMA 2005;293:3022-8

19 Tornos MP, Olona M, Permanyer-Miralda G et al. Is the clinical spectrum and prognosis of native valve infective endocarditis in non-addicts changing? Eur Heart J 1995;16:1686-91.

20 Heiro M, Nikoskelainen J, Hartiala JJ, et al. Diagnosis of infective endocarditis: sensitivity of the Duke vs von Reyn criteria. Arch Intern Med 1998; 158:18-24.

21 Heiro M, Nikoskelainen J, Engblom E, et al. Neurologic manifestations of infective endocarditis: a 17-year experience in a teaching hospital in Finland. Arch Intern Med 2000; 160:2781-7.

22 Heiro $M$, Helenius $H$, Sundell J, et al. Utility of serum C-reactive protein in assessing the outcome of infective endocarditis. Eur Heart $J$ 2005;26:1873-81.

23 Durack DT, Lukes AS, Bright DK, and the Duke Endocarditis Service. New criteria for diagnosis of infective endocarditis: utilization of specific echocardiographic findings. Am J Med 1994:96:200-9.

24 Hoen B, Alla F, Selton-Suty C, et al. Changing profile of infective endocarditis: results of a 1-year survey in France. JAMA 2002;288:75-81.

25 Delahaye F, Goulet V, Lacassin F, et al. Characteristics of infective endocarditis in France 1991: a one-year survey. Eur Heart $J$ $1995 ; 16: 394-401$

26 Fefer $\mathbf{P}$, Raveh D, Rudensky B, et al. Changing epidemiology of infective endocarditis: a retrospective survey of 108 cases, 1990-1999. Eur J Clin Microbiol Infect Dis 2002;21:432-7.

27 Nissen H, Nielsen PF, Frederiksen M, et al. Native valve infective endocarditis in the general population: a 10-year survey of the clinical picture during the 1980s. Eur Heart J 1992;13:872-7.

28 Sandre RM, Shafran SD. Infective endocarditis: review of 135 cases over 9 years. Clin Infect Dis 1996;22:276-86.

29 Van der Meer JTM, Thompson J, Valkenburg HA, et al. Epidemiology of bacterial endocarditis in the Netherlands. I. Patient characteristics. Arch Intern Med 1992;152:1863-8.

30 Steckelberg JM, Wilson WR. Risk factors for infective endocarditis. Infect Dis Clin North Am 1993;7:9-19.

31 Alestig K, Hogevik H, Olaison L. Infective endocarditis: a diagnostic and therapeutic challenge for the new millennium. Scand J Infect Dis 2000;32:343-56.

32 Steckelberg JM, Melton $\sqcup$, Ilstrup DM, et al. Influence of referral bias on the apparent clinical spectrum of infective endocarditis. Am J Med 1990;88:582-8.

\section{IMAGES IN CARDIOLOGY}

\section{Simultaneous combined interventional percutaneous left atrial auricle and atrial septal defect closure}

A 79-year-old man presented with atrial fibrillation, cerebral embolic events and intracerebral bleeding under oral anticoagulation and a haemodynamically significant secundum atrial septal defect (ASD) for percutaneous closure of the left atrial auricle (LAA). Simultaneous interventional therapy was chosen for both sites.

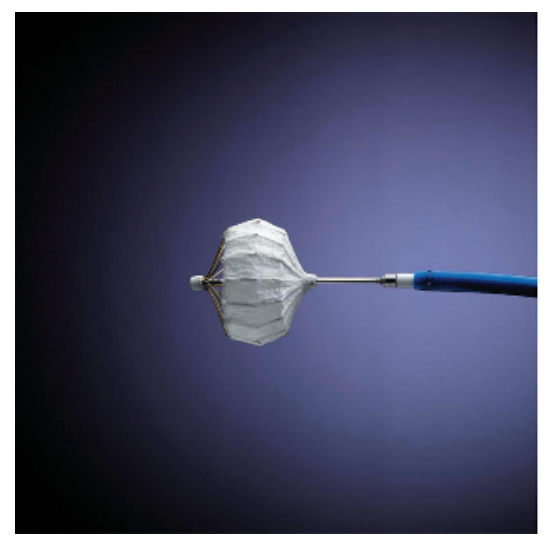

The PLAATO system.
First, the LAA was seeded using the PLAATO system (ev3, Minnesota, USA), then the ASD was closed with an $15 \mathrm{~mm}$ Amplatzer septal occluder system (AGA Medical Corporation, Minnesota, USA). The whole procedure was completed in 1.5 hours; the postprocedural course was uneventful and the patient was discharged the next day.
Percutaneous LAA and ASD closure is a non-surgical alternative in patients with this combination of conditions.

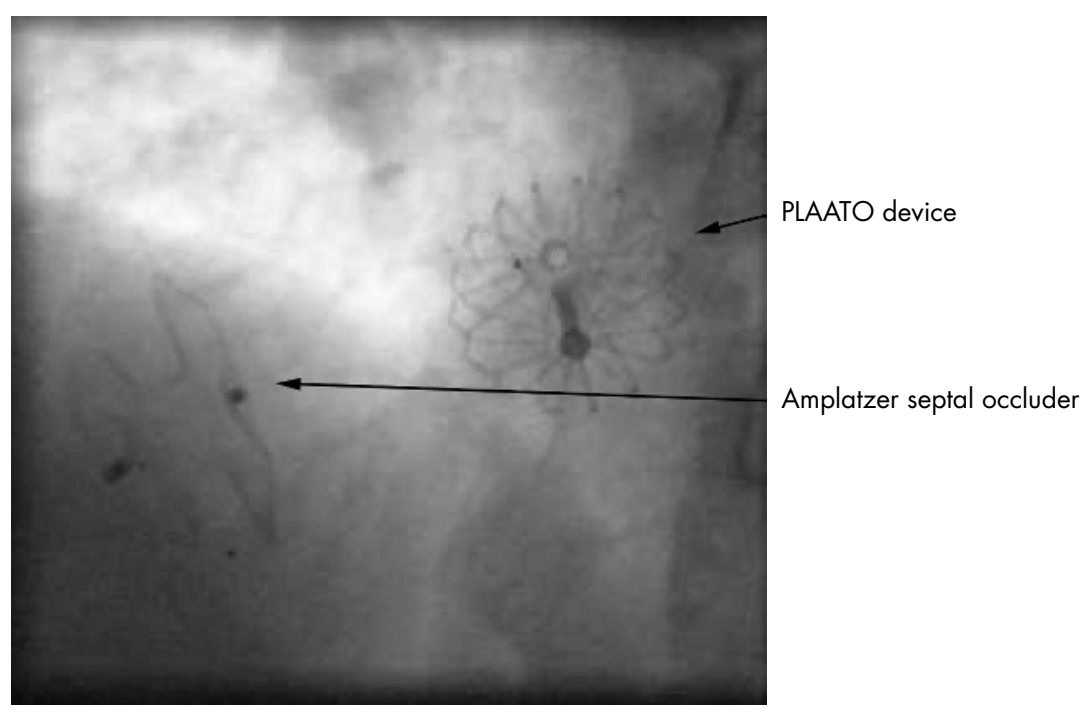

